\title{
Method of evaluating donor corneae for transplantation
}

\author{
EMIL S. SHERRARD \\ Pocklington Eye Transplantation Research Unit, Institute of Opht.halmology, University of London
}

A favourable prognosis for a penetrating corneal graft is largely dependent upon the integrity of the endothelium of the donor cornea, so that any method which will allow the condition of this delicate cell layer to be evaluated in potential transplant material will assist in promoting success.

At present three such methods are in limited use:

(I) The histochemical method of Robbins, Capella, and Kaufman ( 1965) and Kaufman, Capella, and Robbins ( I966) employs staining with para nitro blue tetrazolium to demonstrate the viability of corneal cells. The procedure kills all cells, and it is therefore necessary to assume that the condition of the corneal endothelium is the same in both eyes of a donor pair at any given time. The endothelium of one cornea is evaluated, and if satisfactory, the graft material is taken from the other.

(2) The permeability test (Stocker, King, Lucas, and Georgiade, I966, 1970) uses the permeability of the endothelial cells to the vital dye trypan blue as an index of cell viability. The method evaluates the endothelium of the cornea actually to be used as the graft, but subjects it to much risk of damage during the manipulation involved.

(3) Direct visual examination of the corneal endothelium through the rest of the cornea of intact donor eyes with high power specular-reflection microscopy (Hoefle, Maurice, and Sibley, 1970) is the most simple and least traumatic method, but it does not necessarily follow that an endothelium which appears 'normal' morphologically is functionally competent, and the converse is true.

This paper reports the results of animal experiments on another method of evaluating donor corneal endothelium, but now from the point of view of its net functional efficiency in maintaining stromal deturgescence, a major requirement of most corneal grafts. The endothelium of the actual transplant material is evaluated, in the intact eye, with no risk of damage to the cells. Concurrently the thickness of swollen corneae is reduced closer to normal, and the morphology of the endothelium can be examined by the technique of Hoefle and others (1970).

The method depends upon the temperature reversal phenomenon of the cornea (Davson, I955; Harris and Nordquist, 1955). When a fresh normal eye is refrigerated to below $10^{\circ} \mathrm{C}$. in a moist environment, as in an orthodox eye bank, its cornea swells, and when rewarmed to physiological temperature the cornea thins down again to normal values. This reversal in corneal thickness in relation to temperature is due to great retardation of the corneal metabolic rate in the cold, resulting in the cessation of the outward going active fluid transport system which is located in the endothelium (Mishima and Kudo, 1967; Maurice, 
1969). This allows fluid, which is continuonsly imbibed by the stromal acidic mucopolysaccharides, to accumulate in the stroma and cause it to swell. Rewarming to body temperature restores the metabolic rate and thus the endothelial pump, which expels the accumulated stromal fluid and then maintains a normal inflow-outflow relationship. Thus the cornea thins to and maintains normal thickness until the aqueous humour becomes depleted and/or toxic, when irreversible swelling ensues.

Hedbys and Mishima ( 1966 ) have shown that the relationship between stromal thickness and water content is linear, so that changes in the thickness of the cornea indicate fluctuations in the balance between fluid inflow - endothelial permeability and outflow - the endothelial pumping mechanism.

Therefore, to show that a swollen cornea has the ability to achieve temperature reversal in physiological conditions, and when evaporation from its surface is prevented, is to demonstrate that its endothelial fluid transport system and permeability are competent to restore and maintain normal stromal deturgescence, and thus clarity.

The following experiments were performed to determine whether or not it is practicable to evaluate the corneal endothelium of donor eyes immediately before cutting a graft for transplantation, by demonstrating their ability or inability to achieve temperature reversal. The aim is to eliminate short-term stored donor corneae with faulty endothelia, which clinical experience indicates still escape detection by other methods.

It was necessary to determine the following:

(I) The shape of the temperature reversal curve (thickness against time) for corneae of non-perfused whole eyes which had been stored for various short periods at $4^{\circ} \mathrm{C}$. in a moist chamber (Experiments A, B, and F).

(2) The limitations of the technique.

(3) The minimum time required before the presence or absence of temperature reversal could be definitely recognized (Experiments A and F).

(4) Whether the test procedure involved (prolonged incubation at $35^{\circ} \mathrm{C}$ ) affected the corneal endothelium adversely (Experiment F).

(5) Whether the results of temperature reversal could be correlated with the results of full-thickness grafts (Experiment F).

\section{Material and Methods}

TEMPERATURE REVERSAL

Throughout the experiments and observations, the specular reflection microscope (Maurice, I968) was used for direct examination of the corneal endothelium and to measure stromal thickness and changes during incubation at body temperature $\left(35^{\circ} \mathrm{C}\right)$ of rabbit eyes which had been stored for various periods in orthodox eye-bank moist chambers at $4^{\circ} \mathrm{C}$. During incubation and measuring, silicone oil (MS 200/rocs) covered the anterior surface of the corneae to prevent evaporation and to serve as an immersion fluid for the microscope objective. A $\times 40$ objective with a $\times 8$ eye piece was used for measuring, and with $a \times 16$ eye piece for examining the endothelium.

\section{KERATOPLASTY}

Young adult Dutch rabbits served as hosts and donors. All grafts were $6 \cdot \mathrm{I} \mathrm{mm}$. and performed under Fluothane anaesthesia (Sherrard, 1966) after the technique of Mueller and Smith (1963). The eyes were left open postoperatively. Most recipients were killed after 7 days, but some, where 
indicated, were left for I month. The clinical clarity of the transplants was judged on the $4^{\text {th }}$ and 6 th postoperative days, i.e. when re-epithelialization was complete and before other factors-suture reaction-embarrassed the graft.

Every cornea was finally sectioned and stained with haematoxylin and eosin, Masson's trichrome, or periodic acid Schiff (PAS) for the histological studies.

\section{Experiments and results}

(A) The corneal thicknesses, ignoring the epithelium, of the freshly enucleated eyes of twelve rabbits were measured and their endothelia examined. The eyes were then chilled to $4{ }^{\circ} \mathrm{C}$. for $\mathrm{I} 8 \mathrm{hrs}$. Their corneal thicknesses were again measured to determine the amount of swelling that had occurred in the cold, and their endothelia were re-examined. The epithelia were now removed and the eyes were incubated at $35^{\circ} \mathrm{C}$. Changes in their corneal thicknesses, as measured at half-hourly intervals, were plotted.

All the corneae showed temperature reversal and the resulting curves were very similar in form although the fresh and cold thicknesses of the corneae were variable. Rarely were the corneae of a pair the same thickness fresh ( 2 pairs), most differing by between 5 and io $\mu$, but some ( 2 pairs) showed a $20 \mu$ difference. Only one pair showed the same degree of swelling at $4{ }^{\circ} \mathrm{C}$., and they were not equal when fresh. The average increase in thickness was $170.7 \mu$. During incubation, thinning occurred at between 20.6 and $27.5 \mu$ (av) per hr for $5 \mathrm{hrs}$ and then slowed before levelling off and swelling after a total time of $8 \mathrm{hrs}$. None actually achieved thinning to its fresh (normal) thickness, but all came within $35 \mu$ of it, and the closest was $20 \mu$.

Examination of the corneal endothelium of the eyes in vitro showed morphological changes which occurred consistently in each cornea depending upon its environmental conditions, i.e. fresh or cold, early or late incubation. The appearances were not entirely consistent with those observed by Hodson ( 1969 ) and will form part of a later communication. Fig. I shows the temperature reversal curves from six unrelated eyes, including the extremes of swelling and thinning.

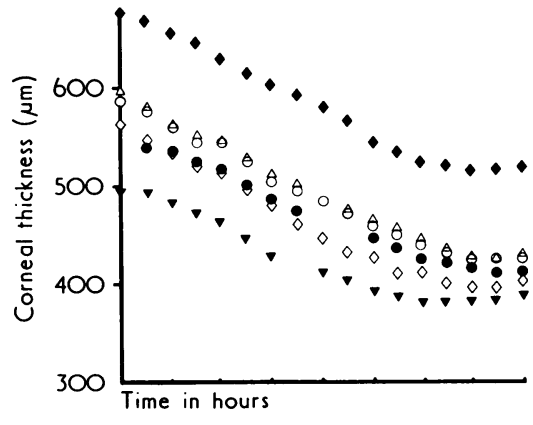

FIG. I Temperature reversal of corneae on rewarming to body temperature after $\mathrm{I} 8 \mathrm{hrs}$ at $4^{\circ} \mathrm{C}$. (Experiments $\left.A\right)$

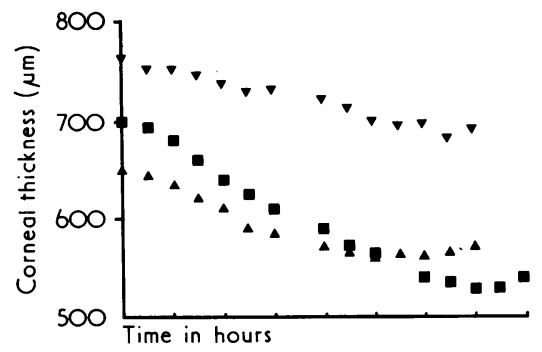

FIG. 2 Temperature reversal of three 5-dayold corneae (Experiments $B$ )

(B) The experiments in $\mathbf{A}$ were repeated upon another six pairs of eyes, but after longer periods of cold storage. Preliminary tests had revealed that most rabbit eyes kept at $4{ }^{\circ} \mathrm{C}$. for 7 days exhibit a flat anterior chamber, disintegration of the iris, and corneal opacity too dense to permit thickness measurements with the specular reflection microscope. There- 
fore, 5 days was considered to be a 'border-line' period of storage. Since the corneae of both eyes of the first three pairs showed temperature reversal (Fig. 2), the time of chilling was extended to 6 days for the remaining three pairs. Even so, five of the corneae thinned on incubation.

Figs 2 and 3 show the resulting temperature reversal curves from one eye of each pair of the 5 and 6-day-old eyes respectively. Apart from their reduced duration, none is dramatically different from the curves obtained from I 8-hr-old eyes, except one ( $\checkmark$ : Fig. 3 ) which maintained its swollen thickness for $6 \frac{1}{2} \mathrm{hrs}$.

None of the corneae thickened initially, indicating an active endothelial pump.

The corneal endothelium during temperature reversal revealed morphological changes similar to those referred to under $\mathbf{A}$.

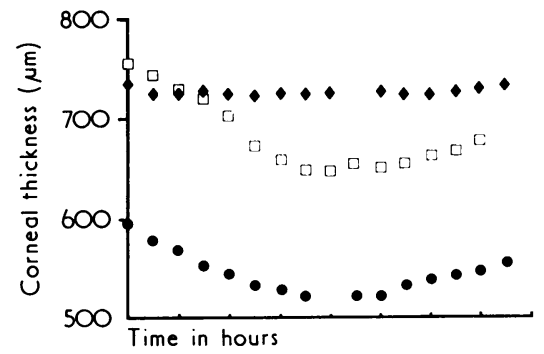

FIG. 3 Temperature reversal of three 6-dayold corneae (Experiments $B$ )

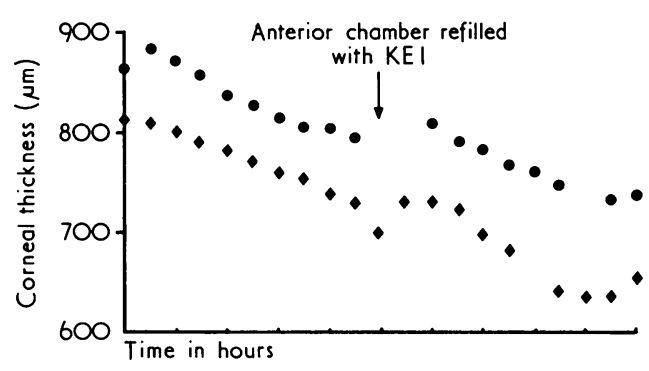

FIG. 4 Temperature reversal of two 6-day-old corneae before and after refilling anterior chamber with $K E I$ (Experiments $C$ )

(C) Four eyes were kept for 6 days at $4^{\circ} \mathrm{C}$. and then incubated at $35^{\circ} \mathrm{C}$. for from 3 to 5 hrs. Their anterior chambers were then evacuated and refilled with an artificial aqueous humour-KEI (Mishima and Kudo, I967). Incubation was continued for a total of ro hrs and half-hourly measurements of the corneal thicknesses were plotted as a graph. The graphs of two of these eyes constitute Fig. 4 and show, in the first stage of incubation, curves similar to those given by the corneae in Experiment B. After replacement of the aqueous humour with KEI, three of the corneae continued to decrease in thickness once having recovered from the manipulations. The total duration of temperature reversal was extended to $9 \mathrm{hrs}$. The cornea of the fourth eye swelled continuously after the introduction of the artificial medium, indicating damage to the endothelium.

(D) The corneal endothelium of both eyes of six freshly killed rabbits was destroyed by rubbing with a bent hypodermic needle inserted through the limbus (Mishima and Hedbys, 1967). A very small amount of aqueous humour was lost from each. The eyes were then enucleated and treated as in Experiment $\mathbf{A}$, except that a very short storage period ( $\mathrm{I} \mathrm{hr}$ ) was used to prevent excessive swelling of the corneae in the absence of their endothelia (Mishima and Hedbys, 1967). On incubation at $35^{\circ} \mathrm{C}$., all the corneae showed instant and rapid swelling. Fig. 5 (overleaf) shows the graphs from two of the eyes.

(E) The eyes were enucleated from six rabbits and placed in the specular reflection microscope substage incubator. Their corneal endothelia were observed while the incubator temperature was steadily raised until distortion of the endothelial cells occurred (at $54^{\circ} \mathrm{C}$.). The temperature was then reduced, and the eyes cooled to and maintained at $35^{\circ} \mathrm{C}$. and kept under normal temperature reversal conditions. Half-hourly plots were made. Fig. 6 (overleaf) shows the graphs from five of these corneae. They all swelled 


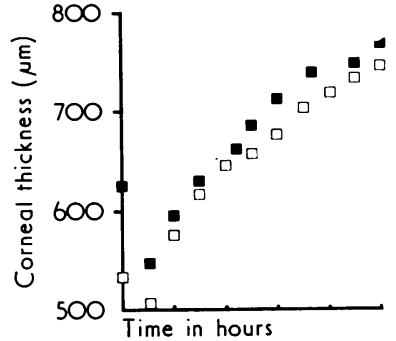

FIG. 5 Swelling curves of two corneae after destruction of endothelia (Experiments D)

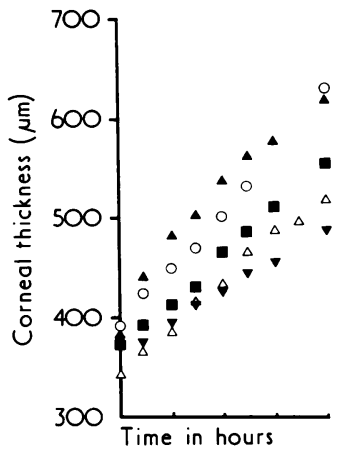

FIG. 6 Swelling curves of corneae after warming to $54^{\circ} \mathrm{C}$. (Experiments $E$ )

rapidly, indicating a non-pumping endothelium and increased permeability. The degenerate appearance of the cells became more accentuated for as long as specular reflection microscopy was possible through the increasingly oedematous stroma, and in some specimens areas of endothelium were seen to detach.

(F) Rabbit eyes were stored at $4^{\circ} \mathrm{C}$. for various periods as follows: six eyes for $24 \mathrm{hrs;}$ three eyes for $48 \mathrm{hrs}$; two eyes for 7 days; one eye for 8 days; and two eyes for 14 days. Four fresh eyes were warmed to $54^{\circ} \mathrm{C}$., and the corneal endothelium was mechanically removed from six others.

Each of the eyes was then incubated at body temperature for 2 hrs, while quarter-hourly measurements of their corneae were recorded. A $6 \cdot 1 \mathrm{~mm}$. full-thickness graft was now taken from each of the corneae after incubation.

The corneae of the eyes stored for 24 and $48 \mathrm{hrs}$ thinned by between 35 and $50 \mu$ during the 2-hr incubation period. The grafts taken from the same corneae were perfectly clear from the first to the sixth postoperative days. Two rabbits with 24 -hr-old grafts, and one with a 2-day-old graft were kept for I month postoperatively, and their transplants were completely clear when they were killed. The endothelium appeared histologically normal in the remains of the donor corneae and all the grafts.

One of the 7-day-old corneae was too thick and hazy for measurement throughout the 2-hr incubation period. The graft taken from it was opaque at surgery but cleared to 95 per cent. transparency by the second postoperative day and so remained until the seventh day. Histologically, the endothelium of the graft appeared normal except for slight leucocyte invasion.

The cornea of the other 7-day-old eye thinned by $60 \mu$ during the 2 hrs of incubation. Visualization of endothial cells was not possible through the stroma which measured 925 to $865 \mu$. Owing to evaporation, the graft cut from this cornea was about 95 per cent. clear at surgery. It gained total transparency on the fourth postoperative day, but like the host, it clouded on the seventh day to reach opacity by the ninth. After suture removal, large areas of transparency returned to the graft, which by the 28th day was clear except for irregular hazy areas around the periphery. The latter were observed histologically to be associated with a digitate retrograft membrane. This was considered to be a successful graft.

The corneae of the eye stored for 8 days swelled by 400 to $800 \mu$ while at $4{ }^{\circ} \mathrm{C}$. It 
maintained this thickness for the first hour of incubation at $35^{\circ} \mathrm{C}$. but thinned by $35 \mu$ during the second hour. Endothelial detail was not observable by specular reflection microscopy. The graft taken from this cornea cleared from 50 per cent. clarity at surgery to 100 per cent. on the sixth postoperative day. This was reduced to 95 per cent. by epithelial oedema on the seventh day.

The cornea of one I4-day-old eye could not be measured. The opaque graft taken from it after 2 hrs incubation cleared to about 90 per cent. transparency on the first postgrafting day. It was yet clearer I day later but then it declined to be 75 per cent. clear on the sixth postoperative day.

The other corneae swelled by $455 \mu$ after the 14 -day storage period, thinned by $60 \mu$ during the 2-hr incubation period, and provided a graft which became ioo per. cent clear on the first postoperative day, from 25 per cent. at surgery, and maintained this clarity until the seventh day, when reaction to the sutures occurred. The transplanted disc returned to complete clarity after suture removal, and was seen at one month to possess a normal endothelium in histological section.

The corneae of the eyes warmed to $54^{\circ} \mathrm{C}$. and those from which the endothelium had been mechanically removed swelled during the $2-\mathrm{hr}$ incubation period. The results of the grafts taken from these corneae are tabulated in Tables I and II.

Table I Donor eyes warmed to $54^{\circ} \mathrm{C}$.

\begin{tabular}{|c|c|c|c|c|c|c|c|c|c|}
\hline \multirow{2}{*}{ Eye no. } & \multicolumn{8}{|c|}{ Per cent. clarity of graft (days postoperatively) } & \multirow{2}{*}{ Result } \\
\hline & $\mathbf{I}$ & 2 & 3 & 4 & 5 & 6 & 7 & 14 & \\
\hline $\mathrm{HI}_{\mathbf{I}}$ & 90 & 95 & 95 & 95 & & 75 & 75 & o & Failed \\
\hline $\mathrm{H}_{2}$ & 95 & 90 & 90 & 90 & & 50 & 30 & $?$ & Failed \\
\hline $\mathrm{H}_{3}$ & 90 & 90 & 100 & 100 & & 90 & 90 & 60 & Successful \\
\hline $\mathrm{H}_{4}$ & $\overline{85}$ & $\overline{90}$ & 95 & 100 & & 100 & 85 & ? & Successful \\
\hline
\end{tabular}

Table II Corneal endothelium removed before incubation

\begin{tabular}{|c|c|c|c|c|c|c|c|c|c|}
\hline \multirow{2}{*}{ Eye no. } & \multicolumn{8}{|c|}{ Per cent. clarity of graft (days postoperatively) } & \multirow{3}{*}{$\begin{array}{l}\text { Result } \\
\text { Failed }\end{array}$} \\
\hline & I & 2 & 3 & 4 & 5 & 6 & 7 & 14 & \\
\hline MI & 95 & 70 & 75 & 70 & & $5^{0}$ & o & 25 & \\
\hline $\mathrm{M}_{2}$ & 90 & 90 & 100 & 95 & & 90 & 95 & 50 & Successful \\
\hline $\mathrm{M}_{3}$ & 80 & 95 & 95 & 95 & 95 & 95 & 100 & 100 & Successful \\
\hline $\mathbf{M}_{4}$ & 95 & 100 & 95 & & 100 & 100 & 95 & 100 & Successful \\
\hline $\mathrm{M}_{5}$ & 90 & 90 & 80 & 75 & & 50 & 0 & 50 & Failed \\
\hline M6 & Ioo & 75 & 50 & 50 & wour & d leak & & & \\
\hline
\end{tabular}

\section{Discussion}

There is apparently no significant difference between the rates of temperature reversal of the corneae of whole unperfused rabbit eyes which have been kept at $4^{\circ} \mathrm{C}$. for from $\mathrm{i} 8 \mathrm{hrs}$ to 
6 days. The duration of reversal, on the other hand, is considerably shortened in the older eyes-by $2 \frac{1}{2}$ to 4 hrs.

Experiment $\mathbf{C}$ indicates that this early cessation of the endothelial pump is a result of depleted and/or toxic aqueous humour, rather than of degeneration of the endothelium, for the pump can be rejuvenated by replacing the remaining aqueous humour with KEI. The works of Riley (personal communication), Hodson (personal communication), and Dikstein and Maurice (1972) support this view.

The early failure of the endothelial pump in the corneae of whole eyes stored for more than 3 days represents one of the limitations of the temperature reversal effect as a test for the integrity of the endothelia of such corneae. It becomes a measurement of the ability of the aqueous humour to support endothelial metabolism rather than a test of the endothelium itself.

A second limitation of the technique as a test of donor corneal endothelium is associated with eyes subjected to longer-term cold storage, in which the stroma becomes very swollen and scatters too much light to permit accurate measurement with the specular reflection microscope. Therefore, it is advisable to reserve the temperature reversal test for corneae of very short-term storage (e.g. I to 3 days). This will expose any faulty corneae which might have been deemed suitable for transplantation by the old criteria.

From the temperature reversal curves obtained for rabbit normal corneae and those subjected to arduous conditions, the indication is that any reduction in thickness of cold swollen corneae, which exceeds $25 \mu$ during $2 \mathrm{hrs}$ of incubation under strict temperature reversal conditions, shows a satisfactory relationship between the fluid outflow and inflow pathways of the endothelium, e.g. the active fluid transport system and the fluid barrier. This means that the endothelial pump is competent to deturgesce the stroma, even if endothelial permeability is above normal.

The combined results of the experiments reported here show that the direction of the curve, e.g. corneal thinning, thickening, and maintenance of thickness, can be prejudged within the first $2 \mathrm{hrs}$ of incubation. Earlier anticipation of the result is untrustworthy as evidenced by one eye ( $\downarrow$, Fig. 3 ), in which the cornea thinned by ro $\mu$ in the first half hour but failed to reduce further, and some of the older corneae in Experiment $\mathbf{F}$. Longer running of the test is unnecessary and leads to excessive depletion of the aqueous humour, with the resulting possibility of endothelial damage at physiological temperatures, especially in older eyes. However, by limiting the use of the test to eyes stored for very short periods, this risk is greatly reduced as demonstrated by the continued thinning of their corneae for a further 5 to 6 hrs.

The ability of corneae to continue the process of temperature reversal after the 2-hr observation period is in itself evidence that the test, e.g. incubation at body temperature for 120 min., has no disastrous effect upon the endothelial pumping mechanism. This is more dramatically shown by the rejuvenation of 6-day-old corneae when the endothelium is supplied with fresh "aqueous humour" resulting in an even larger continuance of temperature reversal than is shown by $\mathrm{i} 8$-hr-old corneae.

The additional advantage of being able to examine the corneal endothelium with the specular reflection microscope immediately reveals any morphological defect of the cell layer in specimens clear enough to transmit sufficient light. Corneae thickened by about $250 \mu$ above normal ( 345 to $420 \mu$ in Dutch rabbits) permit an extremely clear image of their endothelia to be formed, but this becomes increasingly masked by stromal haze until, at about $300 \mu$ in excess of normal, no details can be distinguished. 


\section{Comment}

It seems from the few experimental findings described in the present paper that, in the rabbit, there is no correlation between the results of the temperature reversal of chilled corneae and of full-thickness keratoplasty in which the graft disc was taken from the tested cornea. Although none of the grafts taken from corneae which exhibited temperature reversal failed, some of the corneae which swelled on incubation - including those from which the endothelium had been removed - provided material which resulted in clinically clear transplants. This observation is considered worthy of deeper investigation, because in the past it has been considered by many that, providing the host eye is normal and the operative procedure sound, the ultimate test for the integrity of the endothelium of a donor cornea is the fate of the full-thickness graft taken from that cornea. Furthermore, this has been used as a criterion upon which the results of experimental preservation of rabbit donor eyes have been judged.

It is believed that the remarkable clarity of corneal grafts known to have a non-functional endothelium is due to evaporation. Normally, evaporation from the cornea is greatly retarded by the tear film, which is retained by the superficial epithelium and replenished as necessary by blinking (Mishima and Maurice, I961; Mishima, 1965; Brown and Mishima, 1966). Full-thickness keratoplasty disturbs the superficial epithelium and thus the stability of the tear film, necessitating more frequent blinking.

The rabbit has a low normal blink rate and blinks more frequently with one eye than with both (Sherrard, unpublished). Furthermore, a freshly grafted rabbit eye is usually held closed only to the host-graft-junction. The graft is insensitive and the combined result is that the operated eye is very rarely blinked in response to the stimulus of drying while the other eye blinks normally - thus allowing prolonged periods of evaporation from the transplant. Work is currently in progress to investigate this argument, but at present it is considered that temperature reversal is a better indication of corneal endothelial integrity than a successful graft in the rabbit.

\section{Summary}

(I) The temperature reversal phenomenon, as measured by the specular reflection microscope, has shown to be of value in testing the integrity of the corneal endothelium of whole, unperfused rabbit eyes, which have been kept in orthodox moist chambers for not more than 3 days. The eyes are warmed to body temperature in the microscope substage water jacket and the corneal thickness is measured at fixed intervals. Corneae which show temperature reversal (thinning) are deemed suitable for keraplasty, especially if no morphological discrepancies are seen with the high power of the microscope.

(2) The temperature reversal curves (thickness against time) of the corneae of whole unperfused rabbit eyes after various periods ( $18 \mathrm{hrs}$ to 6 days) of storage at $4{ }^{\circ} \mathrm{C}$. have been established, and the similarity in the rate of reversal noted.

(3) It has been shown that the replacement of the aqueous humour with KEI in rabbit eyes after 5 and 6 days' storage at $4^{\circ} \mathrm{C}$. prolongs the duration of temperature reversal of the corneae, thus bringing them closer to normal thickness, but does not influence the rate of thinning.

(4) The results of temperature reversal can be forecast after 2 hrs' incubation.

(5) Incubation of whole eyes at body temperature is not detrimental to the corneal tissue unless maintained for prolonged periods, i.e. when reversal ceases. 
(6) Penetrating keratoplasties done in parallel with the temperature reversal test do not give parallel results - many corneae which failed to achieve temperature reversal provided material which resulted in clinically clear corneal transplants.

(7) The clarity of rabbit corneal grafts known to have non-functional endothelia is probably due to evaporation permitted by the unilateral blinking habits of this animal.

(8) Doubt is cast upon the validity of the use of results of full-thickness corneal grafts as a criterion for the integrity of donor material in the rabbit.

I am grateful to Prof. Barrie R. Jones and Mr. N. S. G. Rice for their constant encouragement and interest in this investigation and to Miss M. Moore for technical assistance.

\section{References}

BRown, s. 1., and mishima, s. (1966) Arch. Ophthal. (Chicago), 76, 702

DAVson, H. (1955) Biochem. F., 59, 24

Dikstein, s., and maUrice, D. м. (1972) J. Physiol. (Lond.), 221, 29

HARRIS, J. E., and NORDQUIST, L. T. (1955) Amer. F. Ophthal., 40, pt. 2, p. 100

HEDBYs, в. o., and мishima, s. (1966) Exp. Eye Res., 5, 221

Hodson, s. (1969) Nature (Lond.), 222, 676

HOEFLE, F. B., MAURICE, D. M., and SIBLEy, R. c. (1970) Arch. Ophthal. (Chicago), 84, 74I

kaupman, H. E., Capella, J. A., and robbins, J. E. (1966) Amer. F. Ophthal., 6r, 835

MAURICE, D. M. (1968) Experientia (Basel), 24, 1094

(1969) "The Cornea and Sclera", in "The Eye", ed. H. Dawson, 2nd ed., vol. 1., p 489.

Academic Press, New York and London

мishima, s. (1965) Arch. Ophthal. (Chicago), 73, 233

and HEDBys, B. о. (1967) Exp. Eye Res., 6, 10

and KuDo, T. (1967) Invest. Ophthal., 6, 329 and maurice, D. M. (1961) Exp. Eye Res., 1, 46

MUELleR, F. O., and SMITH, A. U. (1963) Ibid., 2, 237

robbins, J. E., Capella, J. A., and Kaufman, H. E. (1965) Arch. Ophthal. (Chicago), 73, 242

SHERRARD, E. (I966) Vet. Rec., 78, 253

STOCKer, F. W., King, E. H., LUGAS, D. O., and Goergiade, N. A. (1966) Arch. Ophthal. (Chicago), 76, 833 